\title{
Ob/ob Mouse
}

National Cancer Institute

\section{Source}

National Cancer Institute. ob/ob Mouse. NCI Thesaurus. Code C122236.

The obese mutant mouse was derived from a spontaneous mutation in a V/Le progenitor mouse at the Jackson Laboratory in 1949. 